\title{
Ginkgo Biloba Extract EGb 761 Alleviates Hepatic Fibrosis and Sinusoidal Microcirculation Disturbance in Patients with Chronic Hepatitis B
}

\author{
Cai Fen Zhang ${ }^{\mathrm{a}}$, Chun Qing Zhang, ${ }^{\mathrm{a}, \mathrm{b}}$, Yu Hua Zhu ${ }^{\mathrm{a}}$, Jing Wang ${ }^{\mathrm{a}}$, Hong Wei Xu', Wan Hua Ren ${ }^{\mathrm{a}}$
}

\begin{abstract}
Background: Few clinical data are available regarding the effect of Ginkgo biloba extract (EGb 761) on liver microcirculation and fibrosis. This randomized, controlled trial is to investigate the effect of Ginko biloba extract EGb 761 on liver fibrosis and hepatic microcirculation in patients with chronic hepatitis B.
\end{abstract}

Methods: Sixty-four patients with chronic hepatitis B were randomized for intention-to-treat. Thirty-two patients were assigned to treated group receiving EGb 761 plus polyunsaturated phosphatidylcholine (Essentiale), 32 patients received Essentiale as controls. Blood samples were taken for measurement of transforming growth factor beta-1 (TGF- $\beta 1$ ), platelet activate factor (PAF), endothelin 1 (ET-1). Twenty-six patients in treated group and 21 patients in control group underwent liver biopsies for histology before and after treatment. Ultrastructural study for sinusoidal microcirculation before and after treatment was carried out on 10 randomly selected patients in each group.

Results: In the treated group, after EGb 761 treatment, there was a significant reduction of blood TGF- $\beta 1$, PAF and ET-1 $(p<0.05)$, whereas this was not observed in the controls. After treatment in both groups, there were significant decrease of ALT, TBil and PT (p $<0.05)$, and significant increase of ALB $(p<0.05)$. Hepatic inflammation and fibrosis significantly alleviated in the treated group, but not in the controls. After EGb 761 treatment, electron microscopy showed red blood cell aggregates and microthrombosis disappeared or decreased in sinusoids; collagen deposits in sinusoidal lumen and Disse space reduced; sinusoidal capillarization alleviated.

Conclusions: EGb 761 can improve sinusoidal microcirculation, alleviate inflammation and inhibit fibrosis through multiple mechanisms, it is effective in the treatment of chronic liver diseases.

Manuscript received September 16, 2008; accepted October 6, 2008

${ }^{a}$ Department of Gastroenterology, Provincial Hospital affiliated to Shan-

dong University, Jinan, China, 250021.

${ }^{\mathrm{b}}$ Corresponding author: zhchqing@medmail.com.cn

doi: $10.4021 / \mathrm{gr} 2008.10 .1220$
Key words: Ginko biloba; EGb 761; Endothelin-1; Hepatic microcirculation; Hepatic fibrosis; Chronic Hepatitis B

\section{Introduction}

Sinusoidal microcirculatory disturbance and liver fibrosis are prominent pathologic features of chronic liver diseases, which are attributable to the progress of chronic hepatitis and development of liver cirrhosis. Therefore, correction of microcirculation disturbance and fibrosis is critical in the treatment of chronic liver diseases. As a therapeutic and cytological protective agent, Ginkgo biloba extract (EGb 761) has been tried in the treatment of cerebral and cardiovascular diseases(1-4), it can improve ischemic microcirculation and tissue impairment. In addition, EGb 761 has also been studied and shown effectiveness in pancreatitis and ischemia intestinal disease in animal models $(5,6)$. Our in vitro study showed that EGb 761 can inhibit hepatic stellate cell (HSC) proliferation and consequently suppress collagen production and extracellular matrix (ECM) secretion(7). Animal study demonstrated that EGb 761 can reverse fibrosis, improve hepatic microcirculation and alleviate hepatocellular damage in fibrotic rats(8), meanwhile severe complications attributable to this treatment were rare. Though EGb 761 has been studied on liver diseases in animal models (8-11), few randomized studies have been reported centering on the EGb 761 efficacy in patients with chronic hepatitis to date. This randomized controlled trial was to investigate the effect of EGb 761 on hepatic fibrosis and microcirculation in patients with chronic hepatitis $\mathrm{B}$, and to investigate the underlying mechanisms.

\section{Patients and methods}

\section{Patients}

Patients with chronic hepatitis B were enrolled in this study. Entry criteria included the following: (1) with hepatitis B history more than 6 months, serum alanine aminotrans- 
ferase (ALT) levels were normal or lower than 3 times the upper limit of normal within 6 months prior to enrolling into the study; (2) serum HBsAg, HBeAg and HBV-DNA were positive; (3) results of liver biopsy within 2 weeks of randomization met histological criteria for chronic hepatitis B; (4) Child-Pugh grading was in A. Exclusion criteria included the concurrent of other active liver diseases as evidenced by hepatitis $\mathrm{C}$, hepatitis A immunoglobulin $\mathrm{M}$ antibody, alcoholic liver disease, or autoimmune liver disease, decompensated liver cirrhosis (ascites, lower extrmeties edema, hepatic coma, variceal hemorrhage ). Patients who had received antiviral, glycocotisone, anti-fibrotic, immunomodulatory therapy within 6 months of randomization were also excluded. Other exclusions included pregnancy, human immunodeficiency virus (HIV) infection, life-threatening illnesses, and inability to provide adequate informed consent.

\section{Study design}

Potential patients were screened at Shandong Provincial Hospital for inclusion and exclusion criteria. Patients were enrolled between May 2003 and June 2006. Patients who met entry criteria provided written informed consent. Consent forms and protocols were approved by the Ethic Review Committee of Shandong Provincial Hospital and were in accordance with the Helsinki Declaration of 1975. After all inclusion/exclusion criteria had been satisfied and consent forms had been signed, patients were randomly assigned to either treated or control group, stratification was not performed. Patients in treated group were given EGb 761 (Willmar Schwabe Pharmaceuticals, Karlsruhe, Germany) 70mg plus polyunsaturated phosphatidylcholine (Essentiale, Aventis pharma Deutschland Gmbh, Cologne, Germany) 930mg intravenously injection daily, for 4 weeks. Patients in control group were given only Essentiale intravenously injection, $930 \mathrm{mg}$ daily for 4 weeks. This study was single blinded to the patients, and the randomization arms were blinded to the pathologists.

\section{Blood samples collection}

All patients were taken $10 \mathrm{ml}$ fasting blood, $5 \mathrm{ml}$ was collected directly into tube without anticoagulant for serum separation, serum was stored in $-20^{\circ} \mathrm{C} ; 3 \mathrm{ml}$ was drawn into a tube containing sodium citrate for prothrombin time (PT) test; $2 \mathrm{ml}$ blood was collected into tube containing $10 \%$ EDTA $\mathrm{Na}_{2} 3 \mu \mathrm{l}$ and aprotinin $40 \mu \mathrm{l}$, centrifuged at $3000 \mathrm{rpm}$, $4^{\circ} \mathrm{C}$, for 10 minutes, supernatant was collected and stored in $-20^{\circ} \mathrm{C}$.

\section{Serum assay}

Automatic biochemical analyzer (Tosoh Corporation, Yamaguchi, Japan) was used to determine blood levels of ALT, albumin (ALB) and total bilirubin (TBil). PT was measured and expressed in seconds.

Enzyme Linked Immunoabsorbance Assay (ELISA) was used to measure serum platelet activate factor (PAF) and transforming growth factor-beta 1 (TGF- $\left.\beta_{1}\right)$. PAF and TGF- $\beta_{1}$ kits were purchased from RapidBio (Dallas, USA) and Genzyme (Dallas, USA) respectively. Serum endothelin-1 (ET-1) was determined using radioimmunoassay (RIA), the kit was purchased from Immuno-Institute (China PLA General Hospital, Beijing, China). All the assay procedures were conducted following the manufacturer's protocols.

Serum HBV DNA titre was measured for each participant at baseline and after treatment, polymerase chain reaction (PCR) method was used for detecting HBV DNA.

\section{Histology}

Liver biopsy specimens were obtained within 2 weeks before randomization and after the completion of treatment. Liver biopsy samples were fixed in $4 \%$ formaldehyde, embedded in paraffin, cut into $4 \mu \mathrm{m}$ thick sections, they were mounted on slides for hematoxylin and eosin (H\&E) staining. The Gordon-Sweet staining was for reticular fibrin, Masson staining was for collagen fibrin.

Severity of liver lesions was graded using the histological activity index (HAI) as described by Knodell et al.(12). HAI inflammatory score and HAI fibrosis score were calculated separately. Liver slide images were automatically collected by Leica DFC480 (Leica Microsystems, Wetzlar, Germany) and IM50 (Leica Microsystems, Wetzlar, Germany) system. Histology examination was carried out by two independent pathologists.

Collagen levels were analyzed using Leica Qwin V3 image analysis program, ten fields of low magnification were randomly observed by the system for each slide, collagen density (percentage of area) in every field was measured, mean value of the percentage was obtained from the 10 fields for each slide.

\section{Ultrastructural study}

Ten pairs of liver biopsy samples from each group before and after treatment were randomly selected for ultrastructural study. These samples were prefixed with $2.5 \%$ gluterodehyde for electron microscopy. They were firstly stored at $4^{\circ} \mathrm{C}$, then were washed with phosphate buffered saline (PBS), post fixed with $1 \%$ osmium teroxide for $1 \mathrm{~h}$ at $4^{\circ} \mathrm{C}$, washed with PBS. A dehydration series of increasing concentrations of ethanol was carried out, then embedded in Epon 812 , cut into $500-700 \AA$ sections, stained with uranyl acetate and lead citrate. Sections were viewed with H-600 transmission electron microscope (Hitachi, Japan). Ten sinusoids randomly chosen were observed for each slide to record the following changes, sinusoidal obstruction and microthrombosis; collagen deposit; capillarization; endothelial cells impairment; ECM deposit in Disse space; impairment of parenchymal cells. A total of 100 sinusoids in each group before and after treatment were observed, and quantitative comparison was conducted. 


\section{Statistics}

Quantitative data were presented as mean $\pm \mathrm{SD}$, categorical variables were reported as percentage values, histological scores of liver biopsies were considered as categorical variables. Baseline features between the two groups of treatment were compared by means of one way of analysis of variances (ANOVA) or Student's t-test for continuous variables. Frequency data were compared using chi-squared test or Fisher's exact test where appropriate. SPSS 11.5 for Windows (SPSS Inc., Chicago, IL, USA) was used for statistical analysis. $p$ value of less than 0.05 was considered statistically significant.

\section{Results}

\section{Baseline Characteristics}

A total of 64 patients were enrolled in the study, they were randomized at 1:1 ratio into the treated group and control group without stratification, 32 patients were assigned to each group. During the study period, 4 patients from control group dropped out, the reasons for dropout were voluntarily discontinuation of the assigned treatment without any adverse effect. Therefore, 60 patients completed the treatment and went into the final analysis (Fig. 1). Twenty-six and 21 patients in treated and control group respectively underwent liver biopsy before and after treatment. Baseline features of the patients in treated and control groups are shown in table
Table 1. Baseline characteristics of the subjects at entry

\begin{tabular}{lccc}
\hline Characteristics & Treated & Controls & p \\
\cline { 1 - 3 } No. patients & 32 & 32 & \\
Age (y) & $44.7 \pm 10.8$ & $42.9 \pm 11.3$ & NS \\
Weight (kg) & $63.7 \pm 11.7$ & $62.2 \pm 9.8$ & NS \\
Male/female & $27 / 13$ & $22 / 12$ & NS \\
Hepatitis history (y) & $3.4 \pm 1.8$ & $3.3 \pm 1.7$ & NS \\
\hline
\end{tabular}

1. The two groups were statistically similar with respect to gender distribution and mean age (Table 1).

\section{Biochemistry and virological response}

After treatment in both groups, blood ALT, TBil, PT decreased significantly $(p<0.05)$, whereas the ALB increased significantly $(p<0.05)$. Either before or after treatment, ALT, TBil, ALB and PT were not significantly different between the two groups $(p>0.05)$. These results indicated the intravenous injection of Essentiale could improve liver function and lower liver enzymes. The Serum HBV DNA titres did not change significantly after treatment in both groups (Table 2).

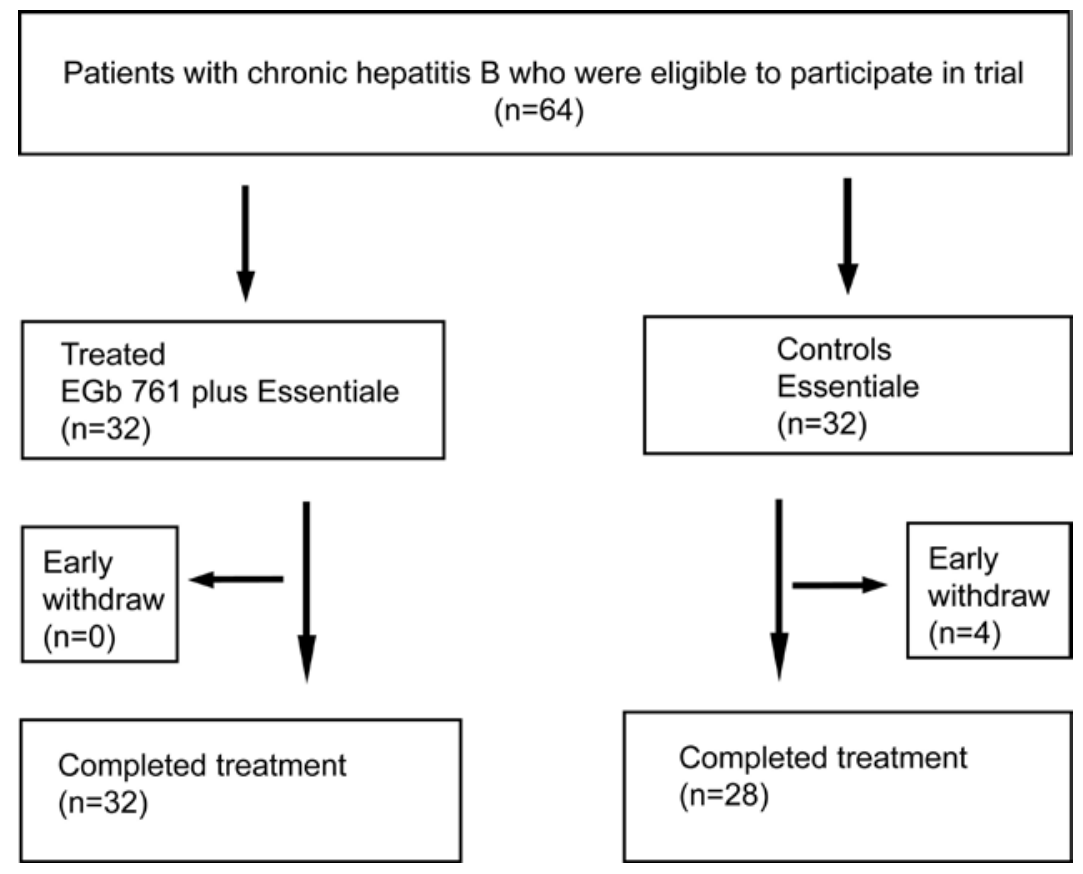

Figure 1. Flowchart of patients included at various stages of the trial. 


\section{Serum TGF- $\beta 1$, PAF, ET-1 levels}

After treatment, TGF- $\beta 1$, PAF and ET- 1 were significantly lower than those before treatment in treated group $(p<0.05)$. Whereas in the control group, these markers did not decrease significantly after treatment $(p>0.05)$. After treatment, these markers in control group were significantly higher than those in treated group $(\mathrm{p}<0.05$, or $\mathrm{p}<0.01)$, (Table 3).

\section{Histological activity index}

Twenty-six patients in treated group and 21 patients in control group underwent liver biopsy for histology before and after treatment. In treated group after treatment, the inflammatory score, fibrosis score and relative collagen levels were significantly lower than those before treatment $(\mathrm{p}<0.05)$, and were also significantly lower than those in the control group after treatment $(\mathrm{p}<0.05)$, (Table 4$)$. Before treatment, we observed swollen hepatocytes, ballooning degeneration, spotty or patchy acidophilic bodies, inflammatory cells infiltration, and red blood cells aggregates in the sinusoids. After treatment, hepatocytes impairment alleviated, acidophilic bodies were rarely seen, with normal hepatic lobular structure, few inflammatory cells infiltration was observed (Fig. $2 a, b)$. Fibrosis and thickened fibrous septa were observed before treatment, whereas after treatment, fibrotic areas reduced significantly, fibrous septa became thinner (Fig. 2c-f).

\section{Sinusoidal ultrastructure}

Before treatment, in both groups, there were hepatocytes necrosis, chondrosome swelling, nuclei shrinkage, hepatic parenchyma was replaced with collagen fibrin (Fig. 3a), microthrombosis presented in 56\% sinusoids (Fig. 3b), different degree of ECM deposit were found in 38\% Disse spaces (Fig. $3 \mathrm{c})$, the sinusoidal endothelial cells damage was observed in $59 \%$ sinusoids. In $32 \%$ sinusoids, endothelial fenestrae decreased or disappeared, with basement membrane formation and sinusoidal capillarization (Fig. 3d). After EGb 761 treatment, parenchymal and endothelial cells damage alleviated, collagen fibrin deposit in sinusoids and Disse spaces decreased, microthrombosis and sinusoidal capillarization also decreased dramatically (Fig. 3e, f), (Table 5).

Table 2. Biochemistry and virology evaluation.

\begin{tabular}{lccccccc}
\hline Group & & $\mathbf{n}$ & $\begin{array}{c}\text { ALT } \\
(\mathbf{I U} / \mathbf{L})\end{array}$ & $\begin{array}{c}\text { ALB } \\
(\mathbf{g} / \mathbf{L})\end{array}$ & $\begin{array}{c}\text { TBIL } \\
(\boldsymbol{\mu m o l} / \mathbf{L})\end{array}$ & $\begin{array}{c}\text { PT } \\
(\mathbf{s})\end{array}$ & $\begin{array}{c}\text { HBV DNA } \\
(\mathbf{x 1 0} \text { copies/ml) }\end{array}$ \\
\hline \multirow{2}{*}{ Treated } & Baseline & 32 & $75.7 \pm 18.5$ & $34.9 \pm 4.4$ & $39.3 \pm 21.2$ & $15.2 \pm 3.4$ & $4.5 \pm 2.7$ \\
& Week 4 & 32 & $37.3 \pm 16.3^{*}$ & $38.2 \pm 5.9^{*}$ & $17.1 \pm 9.5^{* *}$ & $13.2 \pm 2.1^{*}$ & $4.4 \pm 2.4$ \\
& Baseline & 28 & $83.3 \pm 11.4$ & $35.1 \pm 4.8$ & $38.2 \pm 25.4$ & $15.0 \pm 3.5$ & $4.3 \pm 2.5$ \\
Controls & Week 4 & 28 & $35.2 \pm 18.6^{*}$ & $38.3 \pm 6.1^{*}$ & $17.6 \pm 8.1^{*}$ & $13.1 \pm 1.9^{*}$ & $4.4 \pm 2.3$ \\
\hline
\end{tabular}

Data are expressed as mean \pm SD. Fisher's exact test, EGb 761 versus Control. NS, not significant $(p>0.05)$. Comparisons of blood levels of ALT, TBil, PT, ALB, and HBV DNA titres between baseline and after treatment in the same group; and comparisons inter-groups. ALT, alanine aminotransferase; TBIL, total bilirubin; ALB, albumin; PT, prothrombin. ${ }^{*} p<0.05$ VS baseline, ${ }^{* *} p<0.01$ VS baseline.

Table 3. Serum TGF- $\beta 1$, PAF, ET-1

\begin{tabular}{|c|c|c|c|c|c|}
\hline Group & & $\mathbf{n}$ & TGF- $\beta_{1}(\mu \mathrm{g} / \mathrm{L})$ & PAF $(\mu \mathrm{g} / \mathrm{L})$ & ET-1 $(\mu \mathrm{g} / \mathrm{L})$ \\
\hline \multirow{2}{*}{ Treated } & Baseline & 32 & $58.43 \pm 11.04$ & $13.23 \pm 9.79$ & $68.13 \pm 21.71$ \\
\hline & Week 4 & 32 & $17.61 \pm 5.06^{*}$ & $7.62 \pm 6.54^{*}$ & $47.61 \pm 15.34^{* *}$ \\
\hline \multirow{2}{*}{ Controls } & Baseline & 28 & $57.69 \pm 10.23$ & $12.44 \pm 9.63$ & $65.46 \pm 20.67$ \\
\hline & Week 4 & 28 & $61.17 \pm 11.45^{\square}$ & $11.65 \pm 8.96$ & $61.17 \pm 16.45$ \\
\hline
\end{tabular}

Comparisons of TGF- $\beta$, PAF and ET- 1 in two groups before and after treatment. TGF- $\beta_{1}$ : transforming growth factor $\beta_{1}$; PAF platelet activate factor; ET-1 endothelin-1. ${ }^{*} p<0.05$ VS baseline; ${ }^{* *} p<0.01$ VS baseline, $p<$ 0.05 VS week 4 in treated group; $p<0.01$ VS week 4 in treated group. 
Table 4. HAl score and collagen levels.

\begin{tabular}{|c|c|c|c|c|c|}
\hline Group & & $\mathbf{n}$ & Inflammation score & Fibrosis score & Collagen (\%) \\
\hline \multirow{2}{*}{ Treated } & Baseline & 26 & $15.7 \pm 6.3$ & $3.2 \pm 0.6$ & $21.6 \pm 9.3$ \\
\hline & Week 4 & 26 & $10.7 \pm 4.8^{*}$ & $2.2 \pm 0.3^{*}$ & $14.7 \pm 6.4^{*}$ \\
\hline \multirow{2}{*}{ Controls } & Baseline & 21 & $15.6 \pm 5.9$ & $3.3 \pm 0.4$ & $22.7 \pm 9.8$ \\
\hline & Week 4 & 21 & $14.2 \pm 6.6$ & $3.1 \pm 0.5^{\square}$ & $21.2 \pm 9.3$ \\
\hline
\end{tabular}

Hepatic inflammation, fibrosis and collagen deposit before and after treatment in two groups. * $p<0.05$ VS baseline; $p<0.05$ VS 4 weeks in treated group. Collagen density was represented by the collagen percentage in each field, data was mean \pm SD.

Table 5. Sinusoidal microcirculation observation (per 100 sinusoids)

\begin{tabular}{|c|c|c|c|c|c|c|}
\hline Group & & $\mathbf{n}$ & Microthrombosis & Collagen & $\begin{array}{c}\text { Endothelial } \\
\text { Damage }\end{array}$ & Capillarization \\
\hline \multirow{2}{*}{ Treated } & Baseline & 100 & 56 & 38 & 59 & 32 \\
\hline & Week 4 & 100 & $32^{* * \square}$ & $23^{* \square}$ & $41^{* \square}$ & $17^{* \square}$ \\
\hline \multirow{2}{*}{ Controls } & Baseline & 100 & 58 & 37 & 61 & 30 \\
\hline & Week 4 & 100 & 53 & 34 & 57 & 29 \\
\hline
\end{tabular}

Ultrastructural observation of sinusoidal microcirculation in the two groups before and after treatment. Microthrombosis, collagen deposit, endothelial damage and capilliarization were examined for each sinusoid, 100 sinusoids were observed for each group before or after treatment. ${ }^{*} p<0.05$ VS baseline, ${ }^{* *} p<0.01$ VS baseline; $\mathrm{p}<0.05$ VS week 4 in control group.

\section{Discussion}

In this study, we found the EGb 761 is effective on hepatic microcirculation disturbance in patients with chronic hepatitis B. After treatment with EGb 761, liver histology was improved evidenced by reduced inflammation and fibrosis score. Red blood cells aggregates and microthrombosis in sinusoids disappeared or significantly decreased, collagen deposit in sinusoids and Disse space decreased. Impairment of sinusoidal endothelial cells and sinusoidal capillarization alleviated. These clinical results are in accordance with those in our animal studies(8). Though Essentiale improved the liver function and reduced blood levels of liver aminotransferase, the improvement of hepatic microcirculatory disturbances in the control group was not observed.

The thrombosis of medium and large portal veins (PVs) and hepatic veins (HVs) frequently occur in cirrhosis, it is an important factor in the progression of cirrhosis(13). Even in the early stages of hepatic fibrosis, there are profound changes in the hepatic microvasculature. As fibrosis progresses to cirrhosis, intrahepatic shunts increase markedly(14). The sinusoidal microcirculation disturbance in chronic hepatitis B is characterized by red blood cells aggregates, microthrombosis, lumen obstruction, and endothelial cells damage, this not only results in the increased sinusoid pressure, hepatic ischemia, hepatocytes hypoxyemia and degeneration, but also activates HSC via various types of cytokines. HSC can produce a large amount of ECM which deposits in hepatic sinusoids and Disse space to form basement membrane, this in turn worsens hepatic sinusoidal microcirculation disturbance, and leads to hepatic ischemia and hypoxemia, consequently accelerates fibrosis.

Previous studies showed certain medicines and approaches can be used in improving hepatic microcirculation disturbance under various circumstances (15-18). Ginkgo biloba was studied for the potential in the treatment of liver disease in recent years. The standardized Ginkgo biloba extract has two main components, $6 \%$ terpenoid and $24 \%$ 
flavonoid (19). Terpenoid can improve microcirculation, inhibit platelet activation factor, lower blood triglycerides and prevent vascular sclerosis. The flavonoid has the effect of immunological modulation, free radicals removal and antioxidation (20-22). For the effectiveness of EGB 761 on improving hepatic microcirculation and alleviating hepatic fibrosis, the following mechanisms might be involved.

Firstly, EGb 761 removes free radicals and protects cells and tissues against oxidative stress. Lipid peroxidation and free radicals induced impairment are the main injury mechanisms of chronic hepatitis B $(19,20)$. Oxidative stress can cause fibrosis formation, sinusoidal endothelial impairment and hepatic microcirculation disturbance. EGB 761 scavenges free radicals and reactive oxygen species, inhibits lipid peroxidation and lowers malondialdehyde (MDA) levels, hence protects liver cells and alleviates fibrosis(23).

Secondly, EGB 761 prevents the pathological elevation of ET. ET is considered the most potent vasoconstriction polypeptides (24), the elevated ET-1 in blood and hepatic tissue is associated with liver cirrhosis and portal hypertension(24, 25). ET-1 causes microvessels constriction and reduces blood infusion. Continuous hepatic ischemia and hypoxemia increase ET-1 synthesis and release which lead to the transformation of activated HSC into constricted HSC, the latter enhances ECM secretion and hepatic fibrosis(25). Endothelin-1 affects hepatic microvascular exchange, presumably by a direct effect on hepatic sinusoidal endothelial cells. The endothelin antagonist lowers portal pressure in vivo, presumably by acting on hepatic stellate cells, and counteracts the microvascular effects of endothelin-1(24). In our previous animal studies, we found that EGB 761 can significantly decrease ET-1 levels in liver tissue(8), the present study showed the serum ET-1 level decreased significantly in patients with chronic hepatitis B after EGB 761 treatment, this implies the EGB 761 is capable of improving hepatic microcirculation and alleviating fibrosis acting as ET-1 antagonist.

Thirdly, EGb 761 antagonizes platelet activating factor (PAF). PAF plays an important role in the development of hepatic fibrosis and cirrhosis by multiple mechanisms(26). Recently, Yang et al. confirmed that the Kupper cells is the main PAF-producing cells, when in liver cirrhosis, the amount of PAF receptors on HSC significantly increased. The activated and proliferated HSCs play major role in liver fibrosis and portal hypertension development $(27,28)$. EGB 761 can competitively antagonize PAF to combine with its receptor on HSC. Our data suggested the serum PAF levels in patients with chronic hepatitis B decreased after EGB 761 treatment. This demonstrated the EGB 761 alleviate liver fibrosis is associated with lowering PAF levels and inhibiting activation of HSC.

Fourthly, EGb 761 can lower blood TGF- $\beta_{1}$. TGF- $\beta_{1}$ is also a potent cytokine to induce liver fibrosis (29) and promote collagen synthesis(30). Bleser PJ et al showed that the
TGF- $\beta_{1}$ expression is increased by HSC and sinusoidal endothelial cells in fibrosis, this implies the sinusoidal endothelial cell play a role in liver fibrosis(31). In vitro and in vivo data demonstrated that the EGB 761 can inhibit expression of TGF- $\beta_{1}$ thus can inhibit HSC activation and expression of ECM, such as type I and III collagen $(7,32,33)$. consequently inhibit fibrosis.

In conclusion, EGb 761 is capable of removing oxygen free radicals, protecting hepatic sinusoidal endothelial cells, inhibiting blood cells aggregates and thrombosis and reducing collagen deposits in sinusoids and Disse space. These results indicate that the EGb 761 can improve hepatic microcirculation disturbance. Severe complications attributable to this treatment were not observed. Therefore, EGb 761 is a potentially new treatment for the chronic hepatitis B.

\section{Acknowledgement}

This study was supported by the Department of Science and Technology of Shandong Province, China. The authors declare no conflict of interests.

\section{References}

1. Vellas B, Andrieu S, Ousset PJ, Ouzid M, MathiexFortunet H. The GuidAge study: Methodological issues. A 5-year double-blind randomized trial of the efficacy of $\mathrm{EGb} 761(\mathrm{R})$ for prevention of Alzheimer disease in patients over 70 with a memory complaint. Neurology 2006;67:S6-S11.

2. Eckert A, Keil U, Scherping I, Hauptmann S, Muller WE. Stabilization of Mitochondrial Membrane Potential and Improvement of Neuronal Energy Metabolism by Ginkgo Biloba Extract EGb 761. Ann N Y Acad Sci 2005;1056:474-485.

3. Diamond BJ, Shiflett SC, Feiwel N, Matheis RJ, Noskin O, Richards JA, Schoenberger NE. Ginkgo biloba extract: mechanisms and clinical indications. Arch Phys Med Rehabil 2000;81:668-678.

4. Varga E, Bodi A, Ferdinandy P, Droy-Lefaix MT, Blasig IE, Tosaki A. The protective effect of EGb 761 in isolated ischemic/reperfused rat hearts: a link between cardiac function and nitric oxide production. J Cardiovasc Pharmacol 1999;34:711-717.

5. Zeybek N, Gorgulu S, Yagci G, Serdar M, Simsek A, Kaymakcioglu N, Deveci S, et al. The effects of gingko biloba extract (EGb 761) on experimental acute pancreatitis. J Surg Res 2003;115:286-293.

6. Pehlivan M, Dalbeler Y, Hazinedaroglu S, Arikan Y, Erkek AB, Gunal O, Turkcapar N, et al. An assessment of the effect of Ginkgo Biloba EGb 761 on ischemia 

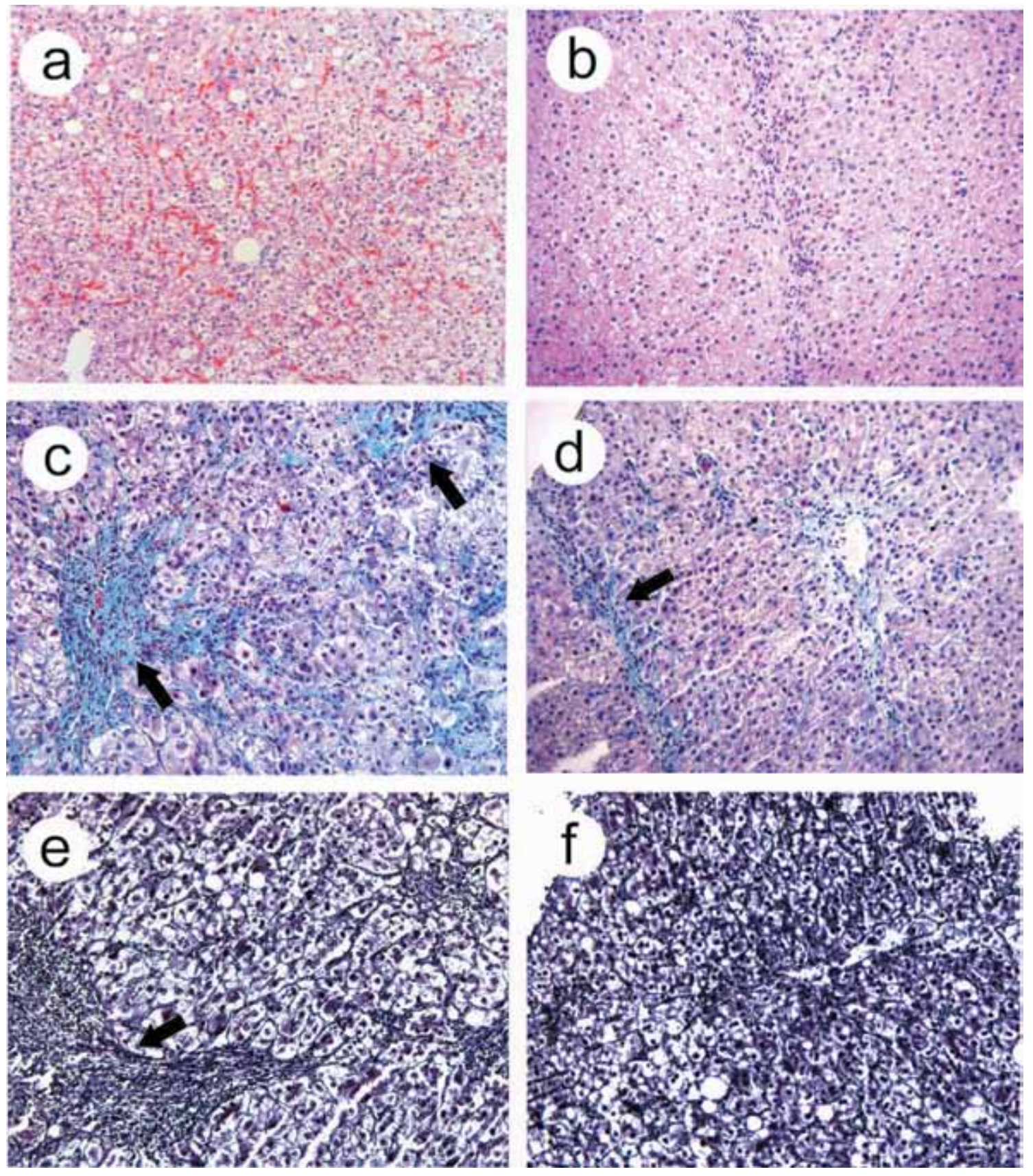

Figure 2. Light microscopic observation after EGb 761 treatment. Light microscopy observations of liver biopsy samples. (a, b), H\&E staining, before treatment, swollen and vacuolated hepatocytes, spotty and patchy acidophilic bodies were seen, inflammatory cells infiltration; aggregates of red blood cells in sinusoids (a); after EGb 761 treatment, acidophilic bodies were rare, no obstructed sinusoids (b). (c, d) Masson 3 staining, collagen fibrin hyperplasia, thickened fibrous septae (arrows, c); after EGb 761 treatment, collagen fibrin decreased, fibrous septa became thinner (arrow, d). (e, f) Meticular fibrin staining, reticular fibrin hyperplasia, fibrous septae thickened (arrow, e); after EGb 761 treatment, reticular fibrin significantly decreased, fibrous septae became thinner (f). Original magnification, a-f, x100. 

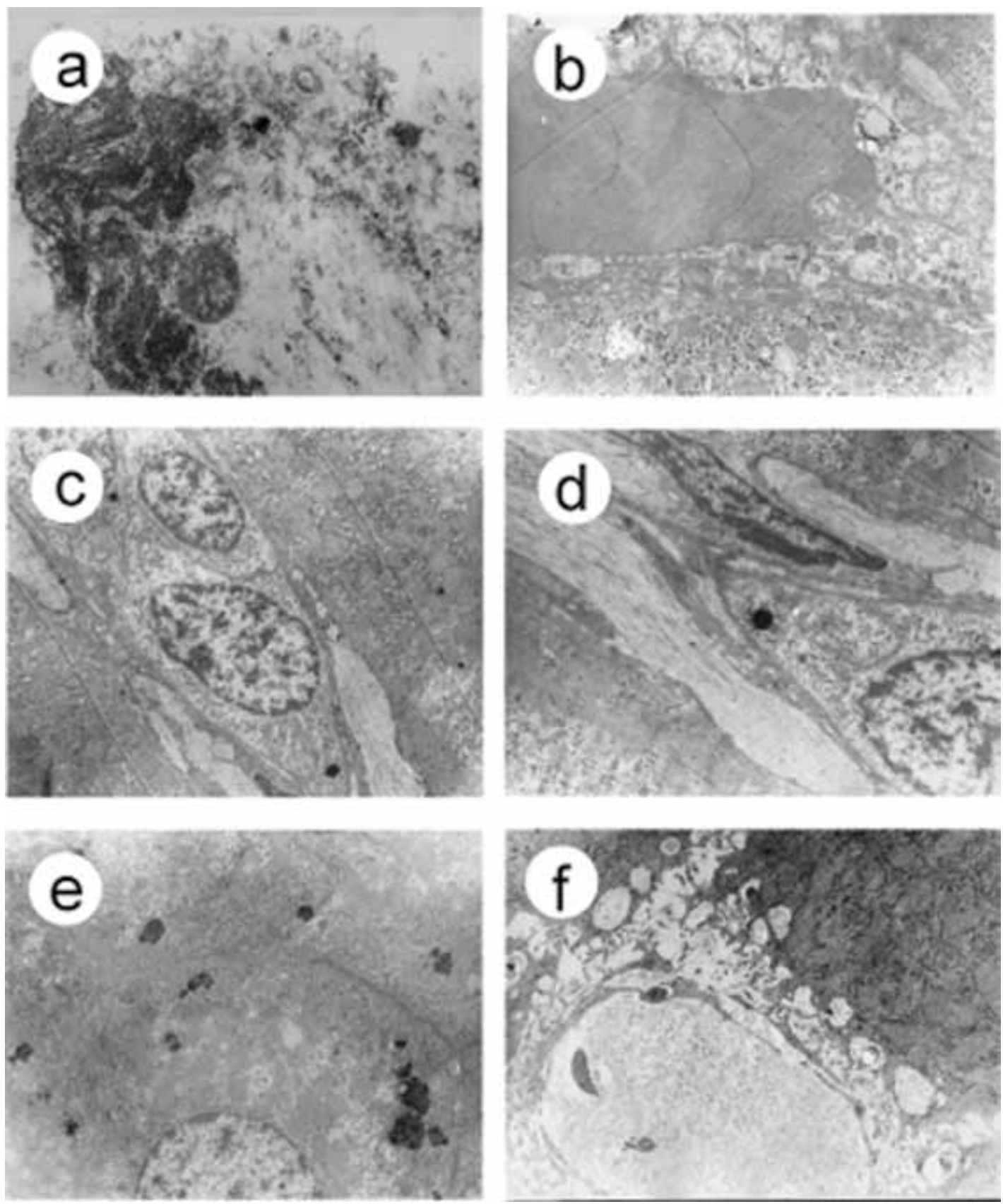

Figure 3. Electron microscopic observation of sinusoids after EGB 761 treatment. (a-d) Before EGb 761 treatment, (a) Hepatocytes lysis, necrosis (arrow), collagen fibrin deposit; (b)mircrothrombosis in the sinusoid (arrow), collagen fibrin deposit in the Disse space; (c) sinusoidal endothelial cells fenestrae disappered, collagen fibrin deposits in the Disse space (arrow) ; (d) collagen fibrin deposit in sinusoids and Disse space (arrow) with clogged sinusoidal lumen. (e-f) After EGb 761 treatment, (e) Hepatocellular organelle degeneration, however cellular morphology, structure and intercellular connection were normal; (f) there was no obstructed sinusoids and Disse space, without microthrombosis and ECM deposits. Original magnification, a-f, $\mathrm{X} 28000$. 
reperfusion injury of intestine. Hepatogastroenterology 2002;49:201-204.

7. Zhang C, Zhu Y, Wan J, Xu H, Shi H, Lu X. Effects of Ginkgo biloba extract on cell proliferation, cytokines and extracellular matrix of hepatic stellate cells. Liver Int 2006;26:1283-1290.

8. Zhang C, Zu J, Shi H, Liu J, Qin C. The effect of Ginkgo biloba extract (EGb 761) on hepatic sinusoidal endothelial cells and hepatic microcirculation in $\mathrm{CCl} 4$ rats. Am J Chin Med 2004;32:21-31.

9. Ding J, Yu J, Wang C, Hu W, Li D, Luo Y, Luo H, et al. Ginkgo biloba extract alleviates liver fibrosis induced by $\mathrm{CCl}$ in rats. Liver Int 2005;25:1224-1232.

10. Topp S, Knoefel WT, Schutte A, Brilloff S, Rogiers X, Gundlach M. Ginkgo biloba (EGB 761) improves microcirculation after warm ischemia of the rat liver. Transplant Proc 2001;33:979-981.

11. Schutte A, Topp SA, Knoefel WT, Brilloff S, Mueller L, Rogiers X, Gundlach M. Influence of Ginkgo Biloba extract (EGB 761) on expression of EGR-1 mRNA and HSP-70 mRNA after warm ischemia in the rat liver. Transplant Proc 2001;33:3724-3725.

12. Knodell RG, Ishak KG, Black WC, Chen TS, Craig R, Kaplowitz N, Kiernan TW, et al. Formulation and application of a numerical scoring system for assessing histological activity in asymptomatic chronic active hepatitis. Hepatology 1981;1:431-435.

13. Wanless IR, Wong F, Blendis LM, Greig P, Heathcote EJ, Levy G. Hepatic and portal vein thrombosis in cirrhosis: possible role in development of parenchymal extinction and portal hypertension. Hepatology 1995;21:12381247.

14. Sherman IA, Pappas SC, Fisher MM. Hepatic microvascular changes associated with development of liver fibrosis and cirrhosis. Am J Physiol 1990;258:H460-465.

15. Ikeda F, Terajima H, Shimahara Y, Kondo T, Yamaoka Y. Reduction of hepatic ischemia/reperfusion-induced injury by a specific ROCK/Rho kinase inhibitor Y-27632. J Surg Res 2003;109:155-160.

16. Stein HJ, Oosthuizen MM, Hinder RA, Lamprechts H. Oxygen free radicals and glutathione in hepatic ischemia/reperfusion injury. J Surg Res 1991;50:398-402.

17. Terajima H, Kondo T, Enders G, Hammer C, Thiery J, Yamamoto Y, Yamaoka Y, et al. Reduction of hepatic microcirculatory failure caused by normothermic ischemia/reperfusion-induced injury by means of heat shock preconditioning. Shock 1999;12:329-334.

18. Koeppel TA, Thies JC, Lehmann T, Gebhard MM, Herfarth C, Otto G, Post S. Improvement of hepatic microhemodynamics by $\mathrm{N}$-acetylcysteine after warm ischemia. Eur Surg Res 1996;28:270-277.

19. Itil T, Martorano D. Natural substances in psychiatry (Ginkgo biloba in dementia). Psychopharmacol Bull 1995;31:147-158.
20. Bridi R, Crossetti FP, Steffen VM, Henriques AT. The antioxidant activity of standardized extract of Ginkgo biloba (EGb 761) in rats. Phytother Res 2001;15:449451.

21. Ergun U, Yurtcu E, Ergun MA. Protective effect of ginkgo biloba against gossypol-induced apoptosis in human lymphocytes. Cell Biol Int 2005;29:717-720.

22. Schindowski K, Leutner S, Kressmann S, Eckert A, Muller WE. Age-related increase of oxidative stressinduced apoptosis in mice prevention by Ginkgo biloba extract (EGb761). J Neural Transm 2001;108:969-978.

23. Aleynik SI, Leo MA, Ma X, Aleynik MK, Lieber CS. Polyenylphosphatidylcholine prevents carbon tetrachloride-induced lipid peroxidation while it attenuates liver fibrosis. J Hepatol 1997;27:554-561.

24. Reichen J, Gerbes AL, Steiner MJ, Sagesser H, Clozel $M$. The effect of endothelin and its antagonist Bosentan on hemodynamics and microvascular exchange in cirrhotic rat liver. J Hepatol 1998;28:1020-1030.

25. Hori N, Wiest R, Groszmann RJ. Enhanced release of nitric oxide in response to changes in flow and shear stress in the superior mesenteric arteries of portal hypertensive rats. Hepatology 1998;28:1467-1473.

26. Kmiec Z. Cooperation of liver cells in health and disease. Adv Anat Embryol Cell Biol 2001;161:IIIXIII, 1-151.

27. Yang Y, Harvey SA, Gandhi CR. Kupffer cells are a major source of increased platelet activating factor in the CCl4-induced cirrhotic rat liver. J Hepatol 2003;39:200207.

28. Yang Y, Nemoto EM, Harvey SA, Subbotin VM, Gandhi CR. Increased hepatic platelet activating factor (PAF) and PAF receptors in carbon tetrachloride induced liver cirrhosis. Gut 2004;53:877-883.

29. Taniguchi H, Kato N, Otsuka M, Goto T, Yoshida H, Shiratori Y, Omata M. Hepatitis C virus core protein upregulates transforming growth factor-beta 1 transcription. J Med Virol 2004;72:52-59.

30. Gressner AM, Weiskirchen R, Breitkopf K, Dooley S. Roles of TGF-beta in hepatic fibrosis. Front Biosci 2002;7:d793-807.

31. De Bleser PJ, Niki T, Rogiers V, Geerts A. Transforming growth factor-beta gene expression in normal and fibrotic rat liver. J Hepatol 1997;26:886-893.

32. Ezquerro IJ, Lasarte JJ, Dotor J, Castilla-Cortazar I, Bustos M, Penuelas I, Blanco G, et al. A synthetic peptide from transforming growth factor beta type III receptor inhibits liver fibrogenesis in rats with carbon tetrachloride liver injury. Cytokine 2003;22:12-20.

33. Breitkopf K, Sawitza I, Westhoff JH, Wickert L, Dooley S, Gressner AM. Thrombospondin 1 acts as a strong promoter of transforming growth factor beta effects via two distinct mechanisms in hepatic stellate cells. Gut 2005;54:673-681. 